\title{
Flow Monitoring in Optofluidic Channels Using Planar Bragg Gratings
}

\author{
C. Holmes, J. C. Gates, C. B. E. Gawith, P. G. R. Smith \\ Optoelectronics Research Centre, University of Southampton, SO17 1BJ, UK \\ chh@orc.soton.ac.uk
}

\begin{abstract}
An integrated pressure/flow sensor using a direct UV written planar Bragg grating situated inside a flexible diaphragm of thickness $200 \mu \mathrm{m}$ has been fabricated. The diaphragm is created by etching away the underlying silicon support.

(C)2009 Optical Society of America

OCIS codes: (130.3120) Integrated Optics Devices; (130.6010) Sensors
\end{abstract}

\section{Introduction}

Mass markets in biotechnology, medical diagnostics, pharmaceutical research and homeland security drive demand for lab on a chip based technology [1]. Flow based sensors form an integral part of these chips for their use as dosing systems and mass flow controllers [2]. This work presents a fully integrated optical microfluidic sensing device that can be combined with high sensitivity evanescent field sensing techniques [3], [4]. From such a device chemical, temperature, pressure and flow rate information of a microfluidic channel can be determined by analysing the reflected/transmitted spectrum from an input broadband source.

A variety of microfluidic flow based sensors are commercially available [5]. An overwhelming amount of these are based on concepts of thermal flow dynamics [6]. One alternative to these thermal flowmeters are ones based on pressure difference flow [5]. Pressure sensors are used to measure flow by sampling the pressure drop along a channel with known fluidic resistance. The advantage they have over thermal flow sensors is that fluid heating is negligible. This can be important when using temperature sensitive fluids or chemical reactions.

The proof of concept device reported has been fabricated from a silicon-on-silica wafer that has a Bragg grating written into it using a technique known as direct UV writing [7]. The Bragg grating is centred over a diaphragm (Figure 1).

(a)

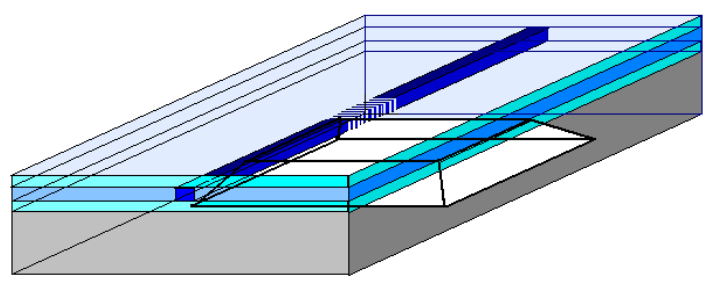

(b)

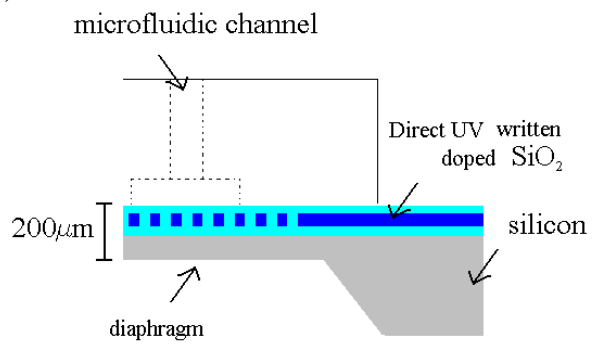

Fig 1. (a)Schematic picture of the flow sensitive Integrated optical chip (b) the partially sectional view in the vicinity of the diaphragm edge, perpendicular to the direction of grating. The dimensions of the diaphragm are $6 \mathrm{~mm} \times 6 \mathrm{~mm} \times 200 \mu \mathrm{m}$.

The diaphragm has a $6 \mathrm{~mm} \times 6 \mathrm{~mm}$ area and a thickness of $200 \mu \mathrm{m}$ that is achieved by anisotropically etching the silicon using $25 \% \mathrm{KOH}$ solution at $70^{\circ} \mathrm{C}$ for 20 hours. A milling process was used to remove the thermal oxide layer present on the bottom of the silicon sample. The remaining thermal oxide acted as a mask when removing the selected silicon in $\mathrm{KOH}$. By back etching the sample the grating becomes much more flexible, inherently increasing its pressure sensitivity.

\section{Methodology}

Our direct UV writing arrangement consists of two focused, overlapping UV laser beams which give an inherent interference pattern that is near-circular and of micron order. Exposure of this intensity pattern into the photosensitive core of a silica-on-silicon wafer results in a change in refractive index. If the beam is left on and translated the intra-spot interference pattern is averaged out, resulting in a channel waveguide. More interestingly, by modulating the writing beam during sample translation channel waveguides with integral index-matched planar 
Bragg gratings can be made [7]. The combination of these two techniques allows planar Bragg gratings to be inserted into complex UV-written devices in a single processing step.

It is understood that stressing a Bragg grating results in both a length and refractive index change. This alters the Bragg condition for the reflected wavelength. This is the concept implemented for pressure monitoring.

\section{Results}

As flow is varied over the diaphragm there is a change in the reflected Bragg wavelength Figure 2 (b). This can be directly related to pressure stressing the grating Figure 2 (a). Applied stress alters the local refractive index and length of grating. Two such diaphragms would be needed in order to obtain a fully functional pressure/flow sensor [8].

(a)

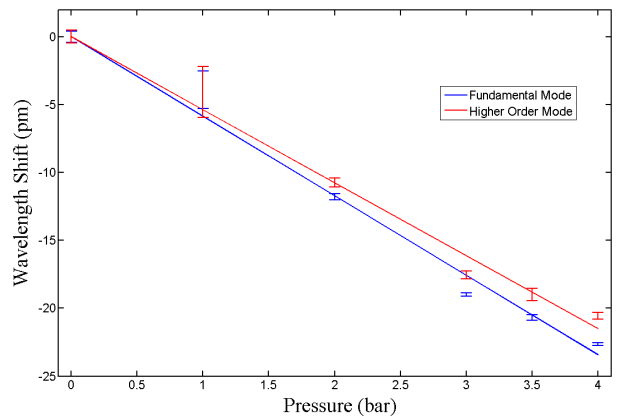

(b)

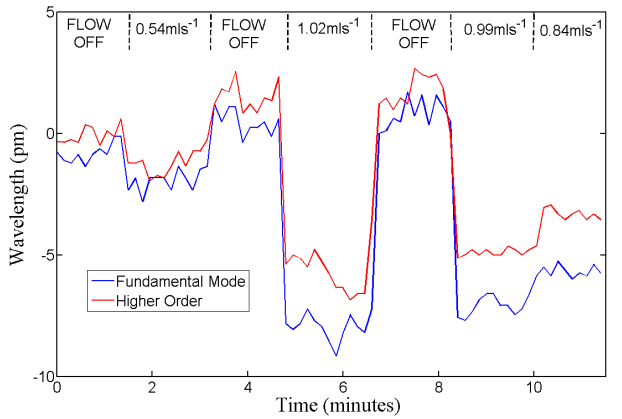

Fig. 2. Bragg wavelength shift for fundamental and higher order modes as function of (a) air pressure and (b) water flow rate varied over time.

From our previous work [3] the effective index of the mode is known to have a temperature sensitivity of $10 \mathrm{pm}^{\circ} \mathrm{C}^{-1}$. This effect can be observed in Figure 2 (b). A temperature reference is common practice in commercially available pressure flow sensors as density and viscosity are strongly influenced by temperature [5]. A reference grating in the bulk can be used to independently monitor temperature deviations.

\section{Conclusion}

We have demonstrated for the first time a back thinned silica-on-silicon Bragg sensor that can be used to create a pressure sensor and flow monitoring device. The data displayed shows a proof of concept flow sensor based on ideas of pressure differential flow sensing. The sensitivity of this device can be improved by further thinning the diaphragm and increasing the diaphragm area. We shall report on the use of a reference grating in order to compensate the thermal effects. We have previously demonstrated microfluidic chemical sensing based on evanescent fields [3]. Integrating this with flow rate monitoring will be performed in the near future. This would allow greater functionality to direct UV written lab-on-a-chip based devices. Such a device would be able to monitor chemical, temperature, pressure and flow rate information of a microfluidic channel by analysing the reflected/transmitted spectrum from an input broadband source.

\section{References}

[1] R Daw, J Finkelstein, Lab on a Chip, Nature 442, 367 (2006)

[2] G M Whitesides, The origins and the future of microfluidics, Nature, 442, 368-373 (2006)

[3] J C Gates et al, New geometry for planar UV written refractive index sensors, SPIE Europe Optics and Optoelectronics, Prague, (2007).

[4] I J G Sparrow, G. D. Emmerson, C. B. E. Gawith, and P. G. R. Smith, "Planar waveguide hygrometer and state sensor demonstrating supercooled water recognition," Sensors And Actuators B-Chemical 107(2), 856-860, (2005)

[5] S Beeby et al, MEMS Mechanical Sensors, Artech House inc., (2004)

[6] M Ashauer et al, Thermal Flow Sensor for liquids and Gases Based on Combinations of Two Principles, Sens. and Act. A, 73, 7-13, (1999)

[7] G. D. Emmerson S. P. Watts et al. Fabrication of directly UV-written channel waveguides with simultaneously defined integral Bragg gratings. Electronics Letters, 38, 1531-1532, 2002.

[8] R E Oosterbroek et al, A micromachined pressure/flow-sensor, Sensors and Actuators A, 77, 167-177, (1999) 